\title{
Cuidado del paciente crónico complejo desde la perspectiva teórica de la comodidad
}

\section{Care of the complex chronic patient from the theoretical perspective of comfort}

\author{
Mónica del Mar Veloza-Gómez ${ }^{1}$; Mónica Rocío Agray-Corredor ${ }^{2}$
}

\begin{abstract}
${ }^{1}$ Enfermera, Esp., M.Sc. Universidad de La Sabana, Facultad de Enfermería y Rehabilitación. Chía - Cundinamarca, Colombia; e-mail: monica.veloza@ unisabana.edu.co; (i) http://orcid.org/0000-0002-0043-2482

²Enfermera, Esp. Clínica Reina Sofía, Jefatura Departamento de Enfermería, Bogotá, Colombia; e-mail: magray@colsanitas.com; (D) https://orcid.org/00000003-0545-010X
\end{abstract}

Cómo citar: Veloza-Gómez, M.D.M.; Agray-Corredor, M.R. 2020. Cuidado del paciente crónico complejo desde la perspectiva teórica de la comodidad. Rev. U.D.C.A Act. \& Div. Cient. 23(1):e1596. http://doi.org/10.31910/rudca.v23.n1.2020.1596

Artículo de acceso abierto publicado por Revista U.D.C.A Actualidad \& Divulgación Científica, bajo una licencia Creative Commons CC BY-NC 4.0

Publicación oficial de la Universidad de Ciencias Aplicadas y Ambientales U.D.C.A, Institución de Educación Superior Acreditada de Alta Calidad por el Ministerio de Educación Nacional.

Recibido: Septiembre 7 de 2019

Aceptado: Junio 12 de 2020

Editado por: Ingeborg Zenner de Polanía

\section{INTRODUCCIÓN}

De acuerdo con la Organización Mundial de la Salud (OMS), las enfermedades crónicas son la principal causa de muerte en el mundo, representando 38 millones (68\%) de los 56 millones de defunciones registradas en 2012. Más del 40\% de ellas (16 millones) fueron muertes prematuras, ocurridas antes de los 70 años (OMS, 2014). De otra parte, están relacionadas con la discapacidad y los años de vida productiva perdidos (AVPP) (Peñaloza Quintero et al. 2014).

La enfermedad crónica es definida como la alteración del funcionamiento normal de la persona, durante un periodo de tiempo, mínimo de seis meses, en el que se vivencia un proceso percibido como amenazante o discapacitante, de naturaleza multicausal, que no puede ser rectificado en forma rápida o fácil y que tiende a la progresión (Röing \& Sanner, 2015; Vargas Escobar, 2017).
Las complicaciones, el dolor y otros síntomas físicos, la dependencia, la soledad, el duelo por lo perdido, las dificultades económicas, los fracasos de los tratamientos y el miedo al futuro, son solo algunos de los eventos que causan en el paciente crónico, una gran carga emocional (Montoya Juárez et al. 2006). Adicionalmente, su plan terapéutico se vuelve cada vez más complejo, aumentando la demanda de cuidados (Martínez Velilla et al. 2018).

Ahora bien, respecto a la condición crónica compleja, hace referencia al conjunto de varias patologías crónicas o una suficientemente grave, con frecuenca, de curso progresivo, asociado a fragilidad funcional, clínica, cognitiva o social, que requiere de un abordaje multidisciplinar y de estrategias de atención integral (Gual et al. 2017).

Para los equipos interprofesionales de atención en salud, el cuidado de estas personas, se constituye en un reto, pues la condición de 
cronicidad compleja requiere de un cuidado integral, del cual, hacen parte las dimensiones física, psicosocial, ambiental y espiritual del ser humano.

El objetivo de este trabajo fue presentar una experiencia relacionada con el cuidado del paciente crónico complejo, identificada a través de la metodología del marco lógico (Muñoz de Rodríguez, 2016), en cuanto a la consideración de la comodidad desde la perspectiva teórica de Kolcaba, y en cuanto al cuidado relacionado con la integridad física, desde el punto de vista de la política institucional y de enfermería de atención humanizada.

\section{MATERIALES Y MÉTODOS}

Mediante la metodología del marco lógico, se identificó la situación problema, representada por las necesidades de comodidad del paciente crónico complejo, hospitalizado en una clínica III nivel de atención, de la Sabana de Bogotá y mediante el planteamiento del objetivo general (brindar cuidado para atender las necesidades de comodidad del paciente crónico complejo) y los objetivos específicos (Atender las necesidades de alivio del paciente crónico complejo; Atender las necesidades de tranquilidad del paciente crónico complejo; Atender las necesidades de transcendencia del paciente crónico complejo), se generó un plan de acción (intervención interprofesional de cuidado), conformado por actividades verificables, a través de indicadores de gestión y de resultado.

La situación problema identificada, se articuló con el planteamiento de la teoría de Kolcaba, en donde la comodidad, como concepto central, se constituye en el valor agregado, tanto para la calidad de vida del paciente y su familia como para la calidad de la atención en salud (Kolcaba \& Kolcaba, 1991; Kolcaba, 2001; Wensley et al. 2017).

Su estructura taxonómica está conformada por los estados y los contextos de comodidad:

Estados de Comodidad. Alivio: satisfacción de una necesidad de comodidad, mediante el control de factores que causan molestia (Kolcaba, 1994; Wilson \& Kolcaba, 2004); tranquilidad: estado de calma o satisfacción, condición necesaria para un rendimiento eficiente (Kolcaba, 1994; Kolcaba et al. 2006); transcendencia: desarrollo de las potencialidades, satisfacción de las necesidades para sobreponerse al dolor y problemas de salud. Lograr independencia y adoptar hábitos saludables (Kolcaba \& Kolcaba 1991; Kolcaba, 1994; Dowd, 2018).

Contextos de Comodidad. Físico: perteneciente a las sensaciones y funciones corporales, incluye todas las dimensiones fisiológicas y homeostáticas de un individuo (Kolcaba et al. 2006); psicoespiritual: perteneciente a la autoestima, el concepto de sí mismo, la sexualidad, el significado en la vida y la relación de uno con un orden o un ser superior (Kolcaba et al. 2006); sociocultural: perteneciente a las relaciones interpersonales, familiares y sociales; ambiental: perteneciente al exterior: entorno, ruido, ambiente, color, temperatura y elementos naturales versus sintéticos (Kolcaba et al. 2006).
Posteriormente y en forma secuencial, se relacionó la Teoría de la Comodidad con la Política de Humanización y la Política del Departamento de Enfermería de la clínica III nivel de atención, de la Sabana de Bogotá.

Política de Humanización. La institución de salud pretende brindar una atención en salud centrada en el paciente y su familia, a través de 4 ejes (Colsanitas, 2016):

1. Brindando acompañamiento y proximidad; 2. Generando ambientes restauradores; 3 . Brindando orientación e información; 4. Construyendo experiencias memorables.

De otra parte, la Política del Departamento de Enfermería propone: "Garantizar cuidados y atención a nuestros usuarios con calidad, calidez, oportunidad, respetando las políticas de ética que rigen nuestra profesión e incentivando acciones en el equipo de trabajo que estén en concordancia con el principio de respeto a la dignidad, velando por la integridad física, espiritual y psíquica de nuestro cliente externo e interno" (Congreso de la República de Colombia, 1996; Colsanitas, 2016).

Mediante la articulación entre la Metodología del Marco Lógico (Muñoz de Rodríguez, 2016), la Teoría de la Comodidad (Kolcaba et al. 2006), la Política de Humanización y la de Enfermería, se generó la intervención interprofesional de cuidado.

Intervención Interprofesional de Cuidado. Esta intervención de cuidado, se desarrolló mediante un proceso conformado por 6 componentes:

1. Ronda de Gestión: Estrategia para la caracterización del paciente crónico complejo, mediante la aplicación de los criterios de identificación (Gual et al. 2017).

2. Ronda ERI (Equipo de Rondas Interprofesionales): Estrategia representada por la revisión de la historia clínica y el desarrollo de la revista interprofesional, con la participación del paciente y la familia, para obtener la información respecto a las condiciones de salud y la identificación de las necesidades de cuidado.

3. Estructura Taxonómica de Kolcaba: Recurso, a través del cual, se clasificaron las necesidades de comodidad.

4. Identificación de las necesidades, de acuerdo con los tipos y los contextos de comodidad, como preámbulo de la intervención interprofesional de cuidado.

5. Modulo Intervención Interprofesional de Cuidado: Componente de la historia clínica institucional, representado por el plan de intervención, acorde a las necesidades de comodidad.

6. Seguimiento a la Intervención Interprofesional de Cuidado: Estrategia de ajuste realizada a través del proceso de recibo y entrega de turno.

La intervención interprofesional de cuidado, se realizó con 5 pacientes; 3 hombres y 2 mujeres, con edades comprendidas entre los 48 a 92 años, quienes cumplieron con los criterios de identificación del paciente crónico complejo (Gual et al. 2017). 


\section{RESULTADOS Y DISCUSIÓN}

Se identificaron 91 necesidades de comodidad, de acuerdo con los tres estados (alivio, tranquilidad, trascendencia) y cuatro contextos (físico, ambiental, psicoespiritual, social), de las cuales, se intervinieron 71 necesidades (Cuadro 1).
En el contexto físico (Brindando Orientación e Información), las intervenciones, en general, se orientaron a favorecer el cuidado de la piel y anexos; la movilidad de los pacientes con limitación, a través del uso de dispositivos; manejo del dolor de manera farmacológica y no farmacológica; adecuación de menús, para favorecer su variedad considerando gustos y necesidades.

Cuadro 1. Necesidades identificadas en los contextos de comodidad.

\begin{tabular}{|c|c|c|}
\hline \multicolumn{3}{|c|}{ TIPOS DE COMODIDAD } \\
\hline ALIVIO & TRANQUILIDAD & TRASCENDENCIA \\
\hline $\begin{array}{l}\text { Dolor,pérdida de la integridad de } \\
\text { la piel, déficit de aseo bucal, } \\
\text { derostomia, ajuste de insulina, } \\
\text { requerimiento de oxígeno, } \\
\text { curación en lesión de miembros } \\
\text { inferior izquierdo, dificultad para } \\
\text { acceso vascular, hipotensión, } \\
\text { edema, retención urinaria, } \\
\text { equimosis en miembros } \\
\text { superiores, ajuste de prótesis, } \\
\text { cuidados de piel, cambios de } \\
\text { posición. }\end{array}$ & $\begin{array}{l}\text { Deterioro de la movilidad, } \\
\text { transtorno de la oxigenación, } \\
\text { disminución del apetito, disnea, } \\
\text { cirugía reciente,inconformidad } \\
\text { con la dieta, alteración en la } \\
\text { perfusión tisular, alteración del } \\
\text { sueño, glicemias no } \\
\text { controladas, alteración de la } \\
\text { memoria. }\end{array}$ & $\begin{array}{l}\text { Necesidada de deambular } \\
\text { independiente, evitar una } \\
\text { posible amputación de miembro } \\
\text { inferior. }\end{array}$ \\
\hline $\begin{array}{l}\text { Necesidad de baño más amplio, } \\
\text { limitación para movilidad, cama } \\
\text { alta, conectividad (Red WIFI). }\end{array}$ & $\begin{array}{l}\text { Riesgo de caída, dificultad para } \\
\text { dormir, limitación para salir de } \\
\text { la habitación, aburrimiento ( falta } \\
\text { de actividades recreativas), } \\
\text { habitación con poco espacio } \\
\text { para deambular con caminador, } \\
\text { entorno poco amable, entorno } \\
\text { desagradable (ruido, luz, } \\
\text { horarios de medicamentos y } \\
\text { actividades). }\end{array}$ & $\begin{array}{l}\text { Necesidad de un entorno } \\
\text { seguro para la deambulación } \\
\text { independiente,adeuación de } \\
\text { casa para cuidados } \\
\text { domiciliarios, necesidad de } \\
\text { regresar a entorno propio } \\
\text { (espacios y objetos propios). }\end{array}$ \\
\hline $\begin{array}{l}\text { Ir a la misa (católica, practicante), } \\
\text { Tristeza, aburrimiento. }\end{array}$ & $\begin{array}{l}\text { Sentimientos de minusvalía, } \\
\text { deseos de morir, depresión, } \\
\text { alteración de la imagen } \\
\text { corporal, temor a ser una carga } \\
\text { para su familia. }\end{array}$ & $\begin{array}{l}\text { Estar en paz consigo mismo y } \\
\text { con Dios, preocupación por } \\
\text { futuro de su hija, pérdida del rol } \\
\text { de padre,incertidumbre por el } \\
\text { pronóstico funcional, duelo } \\
\text { Reciente, ausencia de } \\
\text { significado de la vida, ser } \\
\text { significativo para alguien, }\end{array}$ \\
\hline $\begin{array}{l}\text { Falta de información sobre } \\
\text { condición actual y pronóstico, } \\
\text { visitas del hijo, acompañamiento } \\
\text { permanente, dependencia para } \\
\text { las actividades básicas de la vida } \\
\text { (ABVD)por dificultad para la } \\
\text { movilización. }\end{array}$ & $\begin{array}{l}\text { Dificultad para la comunicación } \\
\text { (traqueostomia), red de apoyo } \\
\text { débil, no quiere ser carga para } \\
\text { sus hijos, cansancio del } \\
\text { cuidador, red de apoyo en duelo } \\
\text { por pérdida reciente }\end{array}$ & $\begin{array}{l}\text { Independencia, preocupación } \\
\text { por la estabilidad laboral, } \\
\text { necesidad de apoyo de otros } \\
\text { familiares de forma } \\
\text { permanente, recuperar rol de } \\
\text { padre, autonomía para la toma } \\
\text { de decisiones sobre su } \\
\text { condición de salud. }\end{array}$ \\
\hline
\end{tabular}

En el contexto ambiental (Generando Ambientes Restauradores), las intervenciones se orientaron en la adecuación de las habitaciones para generar proximidad entre los diferentes elementos considerados, como necesarios, para la comodidad, por parte del paciente (gafas, almohadas, control remoto, televisión, portátil, teléfono); se ayudó y se motivó para la deambulación fuera de la habitación; se realizó cambio de habitación para controlar condiciones de ruido, iluminación y temperatura, de acuerdo con la situación.

En el contexto psicoespiritual (Brindando Acompañamiento y Proximidad), el acercamiento y el interés por parte de los integrantes del equipo interprofesional hacia el paciente, favoreció la relación 
terapéutica, generando espacios para la expresión de emociones, de sentimientos, de necesidades y de preocupaciones.

En el contexto social (Construyendo Experiencias Memorables), la intervención se centró en los cuidadores ofreciendo, mediante la red de apoyo familiar e institucional, acompañamiento, para favorecer su autocuidado (comidas a tiempo, descanso oportuno, actividades recreativas, apoyo espiritual). En pacientes que aún laboraban, se mantuvieron las conexiones laborales, a través del teletrabajo.

Es así como el cuidado del paciente crónico complejo puede cambiar su paradigma tradicional lineal, al considerar intervenciones interprofesionales, respaldadas en la sincronía entre teorías del cuidado - Teoría de la Comodidad (Kolcaba \& Kolcaba, 1991; Kolcaba, 2001), políticas institucionales - Política Institucional de Humanización (Colsanitas, 2016), Política del Departamento de Enfermería y la Metodología del Marco Lógico (Muñoz de Rodríguez, 2016), que propendan por intervenciones de cuidado no convencionales, incluyendo, por ejemplo: menús personalizados, interacción con mascotas, visitas extendidas, apoyo espiritual, apoyo en comunicación, de acuerdo a la cultura institucional, deambulación fuera del entorno específico de cuidado, ambientes de cuidado personalizados y otros, en pro de la comodidad del paciente crónico complejo y propendan por el fortalecimiento de la relación, entre la triada investigación, teoría y práctica.

Existen evidencias, en su mayoría, representadas por estudios descriptivos transversales, mediante la aplicación del instrumento de la teoría y otras evidencias relacionadas con la aplicación de la teoría, que complementan y respaldan los hallazgos de este estudio, en cuanto a los beneficios, desde una perspectiva micro, relacionados con intervenciones de cuidado, basados en la teoría de la comodidad y su impacto a nivel de la integridad física, psíquica y espiritual del paciente crónico y su familia y desde una perspectiva macro, en cuanto a los beneficios para la dinámica de las políticas institucionales de cuidado.

Se evidenció una oportunidad de mejora en cuanto al cuidado relacionado con la integridad espiritual y psíquica y, por lo tanto, con el enfoque integral del cuidado. El cuidado al paciente crónico complejo fue posible, mediante la previa identificación de la situación problema y la posterior aplicación de la Teoría de la Comodidad (Kolcaba, 2001) que, en conjunto con la Política Institucional de Atención Humanizada (Colsanitas, 2016), permitió proponer una intervención de cuidado interprofesional no convencional.

Agradecimientos: Se agradece al Comité de Ética en Investigación de la Fundación Universitaria Sanitas, por su apoyo para el desarrollo del proyecto de investigación, del cual, se derivó este reporte de caso. Conflictos de intereses: El manuscrito fue preparado y revisado con la participación de todos los autores, quienes declaramos que no existe ningún conflicto de intereses que ponga en riesgo la validez de los resultados presentados.

\section{REFERENCIAS}

1. COLSANITAS. 2016. Direccionamiento Estratégico. (Colombia). 26p.

2. CONGRESO DE LA REPÚBLICA DE COLOMBIA. 1996. Ley 911 de 2004. Diario oficial 42710.1996. (Colombia). Disponible desde Internet en: http://www.mineducacion. gov.co/1621/articles-05002_archivo_pdf.pdf (con acceso el 06/10/2019).

3. DOWD, T. 2018. Teoría del confort. En: Alligood, M.R. (ed). Modelos y Teorías en Enfermería. Ed. Elsevier (España). p.527-539.

4. GUAL, N.; YUSTE-FONT, A.; ENFEDAQUE-MONTES, B.; BLAY-PUEYO, C.; MARTÍN-ALVAREZ, R.; INZITARI, M. 2017. Perfil y evolución de pacientes crónicos complejos en una unidad de subagudos. Aten Primaria. (España). 49(9):510-517. https://doi.org/10.1016/j. aprim.2016.11.010

5. KOLCABA, K. 1994. A theory of holistic comfort for nursing. J Adv Nurs. (Estados Unidos). 19:1178-84. https://doi. org/10.1111/j.1365-2648.1994.tb01202.x

6. KOLCABA, K. 2001. Evolution of the mid range theory of comfort for outcomes research. Nurs Outlook. (Estados Unidos).49(2):86-92. https://doi.org/10.1067/ mno.2001.110268

7. KOLCABA, K.; KOLCABA, R. 1991. An analysis of the concept of comfort. J Adv Nurs. (Estados Unidos). 16(11):130110. http://doi.wiley.com/10.1111/j.1365-2648.1991. tb01558.x

8. KOLCABA, K.; TILTON, C.; DROUIN, C. 2006. Comfort theory: A unifying framework to enhance the practice environment. J. Nursing Administration. (Estados Unidos). 36(11):538-544. https://doi.org/10.1097/00005110200611000-00010

9. MARTÍNEZ-VELILLA, N.; IBARROLA-GUILLÉN, C.; FERNÁNDEZ- NAVASCUÉS, A.; LAFITA-TEJEDOR, J. 2018. The funtionality concept as an example of the change in the traditional classification of diseases model. Aten Primaria. (España). 50(1):65-66. https://doi. org/10.1016/j.aprim.2017.03.013

10. MONTOYA-JUÁREZ, R.; SCHMIDT-RÍO-VALLE, J.; PRADOS-PENA, D. 2006. En busca de una definición transcultural de sufrimiento; una revisión bibliográfica. Cultura de los Cuidados. (España).10(20):117-120. https:// doi.org/10.14198/cuid.2006.20.16 
11. MUÑOZ-DE RODRIGUEZ, L. 2016. El Marco lógico en la aplicación de las teorías de rango medio. En: MorenoFergusson, M.E.; Muñoz-De Rodríguez, L. (eds). De la teoría de enfermería a la práctica. Ed. Universidad de La Sabana (Chía). p. 29-42.

12. ORGANIZACIÓN MUNDIAL DE LA SALUD, OMS. 2014. Informe sobre la situación mundial de las enfermedades no transmisibles 2014. Disponible desde Internet en: https:/ / www.who.int/nmh/publications/ncd-status-report-2014/ es/ (con acceso el 10/10/2019).

13. PEÑALOZA-QUINTERO, R.E.; SALAMANCA-BALEN, N.; RODRIGUEZ-HERNANDEZ, J.M.; RODRIGUEZGARCIA, J.; BELTRÁN-VILLEGAS, A.R. 2014. Estimación de la carga de la enfermedad en Colombia, 2010. Ed. Pontificia Universidad Javeriana (Bogotá). p. 73-83.

14. RÖING, M.; SANNER, M. 2015. A meta-ethnographic synthesis on phenomenographic studies of patients' experiences of chronic illness. Int. J. Qual. Stud. Health
Well-being. (Reino Unido). 10(1):1-16. http://dx.doi. org/10.3402/qhw.v10.26279

15. VARGAS-ESCOBAR, L.M. 2017. Intervención educativa para enfermería: cuidado espiritual durante la enfermedad crónica. Aquichan. (Colombia). 17(1):30-41. https://doi. org/10.5294/aqui.2017.17.1.4

16. WENSLEY, C; BOTTTI, M.; MCKILLOP, A.; MERRY, A.F. 2017. A framework of comfort for practice: An integrative review identifying the multiple influences on patients' experience of comfort in healthcare settings. Int J Qual Heal Care. (Reino Unido). 29(2):151-62. https://doi. org/10.1093/intqhc/mzw158

17. WILSON, L.; KOLCABA, K. 2004. Practical application of comfort theory in the perianesthesia setting. J Perianesthesia Nurs. (Estados Unidos). 19(3):164-73. https://doi.org/10.1016/j.jopan.2004.03.006 